\title{
Um Paradigma para Diferenciar o Uso de Memória Implícita e Explícita
}

\author{
A Paradigm to Differenciate Use of Implicit and Explicit Memory
}

\author{
Sabine Pompéia \& Orlando F. Amodeo Bueno* \\ Universidade Federal de São Paulo
}

\begin{abstract}
Resumo
O estudo da contribuição de diferentes subtipos de memória de longo-prazo, explícitas e implícitas, e a contribuição dessa em determinados testes é dificultado por três fatores principais: a) a definição ambígua desses subtipos de memória, "conscientemente" e "não conscientemente" evocáveis, respectivamente; b) as múltiplas características distintas de testes de avaliação de memória implícita e explícita, o que torna pouco reveladora a comparação de seus resultados; e c) o fato de que ambas estratégias de memória podem ser empregadas conjuntamente na realização de praticamente todo tipo de teste. O "Critério de Recordação Intencional" (Retrieval Intentionality Criterion; Schacter, Bowers e Booker, 1989) consiste num paradigma que propõe controlar esses fatores de modo que se possa distinguir a contribuição de memória explícita da implícita. No presente trabalho ilustra-se como empregar essa metodologia e interpretar os resultados advindos de sua aplicação usando o teste de complementação de conjuntos de três letras (stem-completion).

Palavras-chave: Memória; implícita; explícita; intenção; consciência; complementação.
\end{abstract}

\begin{abstract}
The study of the contribution of different subtypes of long-term memory, explicit and implicit, in the performance in certain tasks is constrained by three different factors: a) the ambiguous definition of these types of memory, "consciously" and "non-consciously" retrievable, respectively; b) the distinct features of tasks that assess implicit and explicit memory, rendering the direct comparison of results in these tasks little revealing; e c) the fact that both implicit and explicit strategies can be employed together in practically all types of tests. The "Retrieval Intentionality Criterion" (Schacter, Bowers, \& Booker, 1989) consists of a paradigm that proposes to control these factors so that the use of explicit and implicit memory can be distinguished. The present work illustrates how this methodology can be employed and how to interpret results obtained applying a stem-completion task.

Keywords: Memory; implicit; explicit; intentionality; awareness; stem-completion.
\end{abstract}

Banido da investigação científica por quase um século, o estudo da consciência hoje ocupa uma posição proeminente nas pesquisas em neurociências cognitivas (Moscovitch, 2000). O melhor exemplo disso é o desenvolvimento das pesquisas em memória humana, nas quais questões relacionadas à "consciência” têm sido essenciais desde o início da década de 1980 (Moscovitch, 2000). Grande parte dessas pesquisas centra-se na distinção entre memórias acompanhadas de "consciência" e as que não o são (Moscovitch, 2000), denominadas, respectivamente, de memórias explícitas/declarativas e memórias implícitas/ não declarativas (ver Graf \& Schacter, 1985; Magila \& Xavier, 1999). Embora fuja do escopo do presente trabalho discutir o que vem ou não a ser a "consciência" ou o "inconsciente” (para ampla discussão, ver Farthing, 1992), é necessário apontar alguns aspectos relativos a esses conceitos que freqüentemente se entremeiam com a caracterização dos subtipos de memória.

Uma das maiores contribuições da psicologia cognitiva foi pôr em evidência o fato que processos "não conscientes"

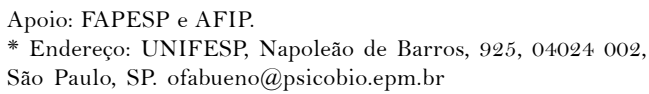

também exibem um importante papel na cognição. Teóricos desse campo de pesquisa mantêm-se céticos, porém, em relação ao conceito psicanalítico de "inconsciente" (Greenwald, 1992). Investigações de processos "não conscientes" são hoje baseadas em áreas como a neuropsicologia e teorias "cognitivo-orientadas" de memória e percepção (Jacoby, Lindsay \& Toth, 1992), que resultaram numa aceitação do "inconsciente cognitivo" como diferindo do inconsciente psicanalítico (Kihlstrom, 1987).

Tendo em vista que não existe uma boa definição dos termos "incosciente" e/ou "inconsciência" (Kihlstrom, Barnhardt \& Tataryn 1992), optou-se, aqui, por utilizar o termo "não consciente" para designar ausência de capacidade de evocar, voluntariamente, informações armazenadas. Em outras palavras, o termo "não consciente" será utilizado quando o input de informações armazenadas na memória afetar o desempenho sem que haja "ciência” de que essas informações foram armazenadas (Farthing, 1992, p.127).

Não parece possível, entretanto, distinguir a memória explícita da "consciência" (capacidade de evocar voluntariamente informações armazenadas), e a memória implícita da "não-consciência”, pois os critérios para demonstrar experimentalmente o processamento "não 
consciente" parecem ser paralelos aos que caracterizam a utilização de memória implícita (Farthing, 1992, p.127). Ou seja, envolvem obtenção de evidências indiretas de memória desprovidas da possibilidade de reportar recordação de eventos (Kihlstrom, 1987; Roediger, 1990; Schacter, 1992). Além disso, o tempo também deve ser considerado nessa discussão quando se considera que para que um teste possa indicar presença de memória é necessário que os sujeitos tenham sido anteriormente expostos às informações a serem lembradas (de forma "consciente" ou não) e que essas informações tenham sido armazenadas: qualquer processo "não consciente" durante a realização de um teste como o de recordação de palavras vistas anteriormente pode ter sido "consciente" por períodos variáveis durante a prévia exposição aos estímulos ou durante a fase de armazenamento dos mesmos na memória (Erdelyi, 1992; Kihlstrom, 1995). Mais ainda, a "ciência" de um fato ou informação pode não ocorrer quando da exposição aos estímulos, tampouco durante o período no qual são armazenados, mas sim durante a realização de um teste, podendo o sujeito pode se dar conta de ter sido exposto àquela informação anteriormente (Farthing, 1992, p.127). Outra questão refere-se aos diferentes tipos de teste de avaliação de memória. Esses podem gerar diferentes medidas de acesso à "consciência”, de modo que o que parece "consciente" numa metodologia pode parecer "não consciente” em outra (Erdelyi, 1992). Enfim, essas restrições parecem limitar tanto o desenvolvimento das pesquisas referentes à memória como as relativas ao papel da “consciência” na cognição.

Tradicionalmente, pesquisas acerca da memória de longo prazo centravam-se no desempenho em testes de avaliação de memória explícita. Ao longo das duas últimas décadas, porém, novas estratégias de avaliação mnemônica foram desenvolvidas de modo a permitir a avaliação de memória implícita, cuja importância como fenômeno a ser estudado só foi largamente aceita após a publicação de um trabalho por Graf e Schacter (1985).

Além da classificação de subtipos de memória de longo prazo em explícita e implícita, essa nomenclatura foi utilizada por muito tempo para também classificar testes que supostamente avaliavam memória explícita e implícita (Richardson-Klavehn \& Bjork, 1988). Assim, a memória explícita (conceito teórico) passou a ser sinônimo do que era observado em testes classificados como de "memória explícita', criando uma espécie de caracterização circular da memória de longo prazo.

Nesse contexto, evidências de dissociação de subtipos de memória explícita e implícita provinham da constatação de diferentes padrões de efeitos em testes explícitos e implícitos, o que chamaremos aqui de abordagem/paradigma clássico. Para avaliação de memória explícita, utilizavam-se testes variados como reconhecimento e recordação livre de estórias, frases ou palavras, enquanto que para a avaliação de memória implícita empregavam-se, preponderantemente, testes de identificação de fragmentos de figuras (ver Pompéia \& Bueno, 1998) ou de complementação de fragmentos ou conjuntos de letras que representam inícios de palavras (ver Pompéia, Paes \& Bueno, 2003).

A presença de prejuízo em testes explícitos e desempenho normal em testes implícitos em pacientes amnésicos era, então, considerada evidência de dissociação de memória implícita e explícita (ver Graf \& Schacter, 1985). Porém, esse paradigma clássico, que propõe contrastar desempenho em diferentes testes considerados como explícitos e implícitos, exibe uma série de limitações. Em primeiro lugar, não está claro o que exatamente diferencia testes explícitos de implícitos. Como apontado por Richardson-Klavehn e Bjork (1988), Erderly (1992) e Farthing (1992, p.127), existe uma ambigüidade na utilização do termo "recordação consciente", que supostamente estaria presente somente em testes explícitos, pois este termo pode descrever dois fenômenos distintos: a) “intenção” voluntária ou deliberada de recordar informações adquiridas anteriormente; ou b) percepção e/ou re-vivência de um episódio desencadeado por pistas fornecidas pelos próprios testes de evocação. Tendo em vista que a re-vivência de um episódio anterior, como a constatação de que uma informação foi previamente vista, não depende do tipo ou da instrução de um teste e pode ou não ocorrer, testes somente poderiam ser definidos como explícitos após a constatação de que envolveram “recordação consciente”. Por esse motivo existe hoje outro tipo de nomenclatura para diferenciar testes de memória que se baseia na presença ou não de intenção voluntária de recordar informações (ver abaixo).

Um segundo fator limitante da abordagem clássica é que ela envolve a comparação de efeitos em testes que avaliam de maneira diferentes a memória explícita e a implícita (Ex.: recordação livre de palavras e identificação de fragmentos de figuras, respectivamente), que não são equivalentes em diversos aspectos. Eles podem diferir em relação a: a) o tipo de estímulo empregado (seguindo esse mesmo exemplo, palavras e figuras); b) o tipo de tarefa de apresentação dos estímulos (ler palavras e avaliar a apreciação de figuras); ou, c) o teste de evocação (recordação livre de palavras, sem pista, por exemplo, ou identificação de fragmentos de figuras vistas, com apresentação de pistas que são os próprios fragmentos). Como esses testes são de naturezas diferentes e envolvem estímulos de tipos distintos, aprendidos de formas dissimilar, o desempenho obtido não é passível de comparação estatística e pode simplesmente refletir diferenças de sensibilidade a alterações mnemônicas (Richardson-Klavehn \& Bjork, 1988). Portanto, a classificação de testes como de memória explícita ou implícita não assegura que os resultados reflitam processos mnemônicos pertencentes a um ou outro tipo de memória.

Um terceiro fator limitante da utilização da abordagem clássica é que não existem testes "puros" de memória de longo prazo, que reflitam somente um ou outro subtipo de memória (Toth, Lindsay \& Jacoby, 1992). O desempenho em testes clássicos envolve utilização conjunta de estratégias implícitas e explícitas, que se somam, embora não seja conhecida a magnitude das contribuições de estratégias explícitas e implícitas no desempenho em cada tipo de tarefa 
(Blaxton, 1989; Richardson-Klavehn \& Bjork, 1988; Roediger, 1990; Toth et al., 1992). Logo, para que a memória de longo prazo seja corretamente avaliada, é necessário encontrar um meio de determinar a contribuição da memória implícita da explícita em cada tipo de teste. Existem hoje novos paradigmas de investigação da memória de longo prazo que procuram resolver as limitações já apontadas dessa abordagem clássica, como será visto a seguir.

Devido à problemática acerca da caracterização de testes como "explícitos" e “implícitos”, a instrução dada no início da tarefa de evocação passou a ser considerada de grande importância na distinção de subtipos de memória avaliados. Johnson e Hasher (1987) propuseram que os testes de memória fossem subdivididos em diretos e indiretos, de acordo com suas instruções. Testes diretos envolvem instruções que solicitam dos sujeitos que recordem informações de um episódio de aprendizado, mencionando o tipo, contex to ou tempo em que o evento ocorreu, como é verificado em testes de reconhecimento, que requerem que os sujeitos apontem estímulos aos quais foram apresentados em seção experimental anterior (Richardson-Klavehn \& Bjork, 1988). Neste caso, a "intenção" de recordar informações aprendidas anteriormente supostamente levaria, pelo menos preponderantemente, à utilização de estratégias explícitas de recordação (Richardson-Klavehn \& Bjork, 1988). Testes indiretos, por sua vez, envolvem atividades motora ou cognitiva relacionadas a um episódio de aprendizado, sem que as instruções se refiram a esses eventos. Isso permitiria minimizar a recordação "intencional" de informações adquiridas anteriormente, o que caracterizaria recordação preponderante de memória implícita (Richardson-Klavehn \& Bjork, 1988).

Baseados na distinção entre testes diretos e indiretos, Schacter, Bowers e Booker (1989) sugeriram uma metodologia que permite dissociar subtipos de memória de longo prazo, o Critério de Recordação Intencional (Retrieval Intentionality Criterion). Esse paradigma experimental envolve dois aspectos principais. Em primeiro lugar, os testes utilizados para avaliar as memórias explícita e implícita devem assemelhar-se em tudo exceto na instrução. Em outras palavras, os testes devem diferir somente no que se refere às instruções, diretas ou indiretas, enquanto que as características dos estímulos-alvo e das pistas externas, que desencadeiam a recordação, devem ser idênticas.

Um bom exemplo envolve o teste de complementação de conjuntos de letras (aqui denominadas tríades) para formar palavras (do inglês stem-completion), como ABO que pode completar palavras como ABÓBORA, teste esse que tem sido utilizado para avaliar uma ampla gama de fatores que influenciam a memória (Graf \& Williams, 1987; Pompéia et al., 2003). Para aplicação do teste de complementação de tríades, é inicialmente necessária uma fase de apresentação dos estímulos que serão utilizados para avaliar a memória (fase de apresentação, codificação, aprendizado, ou fase de estudo). Subseqüentemente, em outra fase (fase teste ou fase de evocação), solicita-se que sejam completadas tríades iniciais de palavras que correspondem às palavras vistas na fase de apresentação. A memória é verificada quando a probabilidade estimada de complementação de uma tríade com uma palavra após essa ter sido apresentada na primeira fase (palavras familiares, ou pré-ativadas) é maior que a probabilidade de complementação com essa mesma palavra ao acaso, isto é, a probabilidade de que essa mesma palavra seja utilizada para completar a tríade sem que o sujeito tenha sido a ela apresentado.

Retomemos a utilização do Critério de Recordação Intencional (CRI) através do uso do teste de complementação de tríades. Embora a instrução clássica desse teste seja "complete com a primeira palavra que vem à mente" (teste indireto), ele também pode ser realizado após instrução direta, tal como "complete com uma palavra que viu". Tendo em vista que o que distingue os testes diretos dos indiretos é a volição, ou intenção, de evocar informações aprendidas anteriormente, fica eliminada, na verificação da memória, a primeira das desvantagens presente na utilização da abordagem clássica, a saber, a ambigüidade relativa ao emprego da "recordação consciente" para distinguir entre testes “explícitos” e "implícitos”.

O CRI propõe,desta forma, a aplicação de testes como esses, exatamente iguais exceto na instrução, após a mesma tarefa de aprendizado de estímulos a serem completados em ambas as tarefas, isto é, codificados com o mesmo nível de processamento (ver Blaxton, 1989; Roediger, 1990), expostos pelo mesmo período de tempo, na mesma ocasião, com o mesmo padrão perceptual (tipo de fonte, por exemplo), etc. Desta forma, elimina-se a segunda desvantagem da abordagem clássica: são comparados testes de avaliação de diferentes tipos de memória que não fazem demandas informacionais distintas, excluindo-se a instrução que direciona a intenção de evocar ou não informações.

O segundo ponto a ser seguido na aplicação do CRI é realizar alguma manipulação que afete seletivamente um dos subtipos de memória como manipulações do tipo/nível de processamento. Sabe-se, por exemplo, que o processamento conceitual/semântico, durante a apresentação de estímulos aumenta a memória explícita para esses estímulos e não afeta a memória implícita (Blaxton, 1989; Richardson-Klavehn \& Bjork, 1988; Roediger, 1990). Assim, é possível abordar a terceira falha da metodologia clássica: pode-se verificar a ocorrência de contaminação de memória implícita em testes de avaliação de memória explícita e vice-versa, pois é esperado um efeito de processamento somente na versão direta dos testes (aumento da recordação quando do processamento mais profundo, ou processamento conceitual/semântico) e ausência desse efeito na versão indireta do teste. Em termos simplificados, então, a verificação de efeito de processamento semântico na tarefa indireta constitui um indício de contaminação de memória explícita, ao passo que a ausência de efeito de processamento em testes diretos indica alguma falha metodológica, que sugere que a memória explícita não está sendo predominantemente empregada.

A aplicação do CRI, portanto, permite a verificação de diferenças estatísticas no desempenho de tarefas diretas e 
indiretas, equiparáveis após manipulação de processamento durante a codificação, que podem indicar a utilização de estratégias distintas de memória em cada tipo de teste (Richardson-Klavehn \& Bjork, 1988; Schacter et al., 1989).

O objetivo do presente trabalho foi empregar o Critério de Recordação Intencional aos resultados obtidos em versões indiretas e diretas do teste de complementação de tríades empregando estímulos adequados para uso em português do Brasil. Optou-se aqui por utilizar testes de complementação de palavras de 5 letras (Ex.: SOL__ para completar palavras como SOLAR, SOLDA, etc.) ao invés de testes de complementação com palavras com número de letras indefinido (Ex.: SOL_____ para completar SOLA, SOLDADO, SOLIDARIEDADE, etc.). O motivo para essa escolha foi o de dificultar o teste, já que a população estudada consistia de sujeitos com alta escolaridade, para quem o teste de complementação com palavras de qualquer tamanho não apresenta desafio algum. Para outros experimentos que usam complementação com palavras de 5 letras, ver Jacoby (1998) e Pompéia (2000). Devido à inexistência de publicações no Brasil de normas de complementação de tríades que completam 5 palavras, foi realizado um estudo piloto para determinar as palavras a serem empregadas no presente experimento (ser seleção de estímulos no método). Normas para a complementação de tríades com palavras de tamanho indefinido que completam pelo menos 10 palavras comuns em português do Brasil podem ser encontrados em Pompéia et al. (2003).

\section{Método}

\section{Participantes}

Particiaparam 12 estudantes universitários saudáveis (dos quais 6 homens) com idade de 24,8 $\pm 5,7$ anos (média $\pm D P$ ) e para quem o português falado no Brasil era a primeira língua.

Seleção de estímulos e construção de listas (Anexo A): para a obtenção de normas para seleção de estímulos foram inicialmente selecionadas, com o auxílio do "Dicionário para Palavras Cruzadas Diretas" (1983), 226 tríades que constituíam o início de duas a 10 palavras de 5 letras. Essas tríades foram listadas em quatro tipos de formulários com tríades em diferentes ordens. Em um estudo piloto, 69 estudantes de medicina (dos quais 39 homens) com idade de 20,4 $\pm 1,1$ anos (média $\pm D P$ ) foram instruídos, conjuntamente durante uma aula, a completar as tríades de um dos formulários "com a primeira palavra que lhes viesse à mente", evitando usar nomes de pessoas e palavras no plural. O limite de tempo para realizar a tarefa foi de uma hora. Os sujeitos foram instruídos a não se ater às tríades que exigissem muito esforço para complementação e a deixálas em branco caso não conseguissem completá-las com relativa facilidade.

Não foram computadas as complementações nos seguintes casos: palavras inexistentes em português, ilegíveis, incorretamente grafadas, nomes de pessoas e palavras no plural.
O padrão de complementação das 226 tríades pode ser encontrado em http://www.unifesp.br/dpsicobio/adap/ adapta.htm. Houve muitos erros e omissões de complementação devido à dificuldade de encontrar palavras para completar as tríades. Desta forma, foram consideradas inadequadas para a tarefa de complementação as 10 tríades que foram completadas por menos de um terço da população estudada (23 dos 69 indivíduos), isto é, as tríades ARI, CAC, DUC, GOL, GUI, PUN, RAM, REC, RED e TAR.

Foram selecionadas 108 palavras de 5 letras com tríades distintas dentre as 216 tríades consideradas adequadas para testes de complementação. Estas palavras foram subdivididas em 9 conjuntos de 12 palavras (conjuntos $\mathrm{A}$, B, C, D, E, F, x, y, z; Anexo). Compilaram-se estes conjuntos balanceando as seguintes características das tríades: a) o posicionamento do estímulo entre demais complementações para sua tríade, isto é, se a palavra era a primeira, a segunda, a terceira mais completada, etc.; b) a probabilidade de complementação ao acaso; c) o número de palavras alternativas completadas para a mesma tríade; e d) o número total de complementações para a tríade. Foram também empregadas 6 palavras de 5 letras começando com tríades diferentes das 108 palavras acima citadas e que também completavam diversas palavras. Estes estímulos foram utilizados para controlar efeitos de primazia e recência (ver Gershberg \& Shimamura, 1994) e foram os mesmos para todas as listas apresentadas.

Foram criadas três listas de apresentação de 36 palavras (listas compostas dos conjuntos $\mathrm{ABx}, \mathrm{CDy}$ e $\mathrm{EFz}$ ) que reuniam: a) três conjuntos de 12 palavras de 5 letras; b) três palavras incluídas ao início e três ao final das listas, para evitar os efeitos de primazia e recência (sua recordação foi desconsiderada). Essas listas não diferiram quanto às características empregadas para balancear os conjuntos [posicionamento: $\mathrm{F}_{2,70}=0,21, p=0,081$; probabilidade de complementação ao acaso: $\mathrm{F}_{2,70}=0,24, p=0,79$; número de palavras alternativas: $\mathrm{F}_{2,70}=0,50, p=0,61$; número total de complementações: $\left.\mathrm{F}_{2,70}=0,04, p=0,96\right]$. Somente duas listas eram apresentadas a cada participantes.

Foram criadas duas listas de recordação que continham 36 tríades que completavam palavras de um conjunto de cada uma das listas de apresentação (tríades dos conjuntos ACE e BDF). Ver procedimento e Tabela 1 para melhor compreender a aplicação dessas listas.

\section{Procedimento}

O experimento foi aprovado pela Comissão de Ética em Pesquisa da Universidade Federal de São Paulo. Os participantes foram avaliados individualmente após assinarem termos de consentimento informado.

Duas tarefas de apresentação/codificação foram realizadas, cada uma durante a apresentação de uma das listas de apresentação: a) apreciação (tarefa de processamento conceitual/semântico), na qual cada palavra era avaliada de acordo com a seguinte escala: gosto, neutra, não gosto; b) contagem de espaços (tarefa de processamento perceptual), 
na qual era contado o número de espaços fechados circunscritos pelas letras que formam cada palavra. Por exemplo, a letra $\mathrm{B}$ circunscreve dois espaços, as letras A, O e R circunscrevem um, e E, I e J não circunscrevem nenhum espaço fechado. Sendo assim, cada sujeito era exposto a duas listas de apresentação.

Após as tarefas de apresentação, os sujeitos foram submetidos a uma atividade distratora, com duração de 3 minutos (teste de substituição de dígitos por símbolos, DSST), seguido imediatamente pelo teste de recordação.

O teste de recordação foi o de complementação de tríades para formar palavras de 5 letras. Esse teste foi realizado com duas instruções distintas, cada uma para uma das três listas de recordação diferentes, na seguinte ordem: a) complementação indireta ("complete com a primeira palavra que vem à mente"); b) complementação direta ("complete com palavras vistas anteriormente durante a apresentação de palavras"). Caso os participantes não recordassem palavras vistas, deveriam completar as tríades com a primeira palavra que lhes viesse à mente. Os sujeitos, portanto, completavam tríades das duas listas de recordação. Os escores foram as proporções de tríades completadas com palavras-alvo (presentes nas listas de codificação).

Em ambos os testes de complementação direto e indireto, os sujeitos tinham a oportunidade de demonstrar sua recordação de palavras familiares, provenientes de um conjunto de estímulos visto na tarefa de apresentação perceptual, e de um conjunto de palavras codificadas conceitualmente (ver Tabela 1). Além disso, complementações referentes a estímulos que correspondiam a conjuntos não vistos pelos sujeitos (estímulos nãofamiliares) foram utilizados para determinar o desempenho ao acaso em cada teste, ou seja, a probabilidade de que palavras-alvo, ou presentes nos conjuntos, fossem utilizadas nos testes de recordação sem que os sujeitos tivessem sido a elas expostos na fase de apresentação. Ou seja, a lista de recordação ACE continha tríades das palavras dos conjuntos $\mathrm{A}$ (da listas de apresentação ABx), C (da lista de apresentação $\mathrm{CDy}$ ), e E (da lista de apresentação EFz). Para cada sujeito um desses conjuntos correspondia a palavras vistas na tarefa de apresentação perceptual, outro, na de apresentação conceitual, e o restante completava palavras não vistas pelo sujeito (palavras não-familiares). A avaliação da complementação de palavras dos conjuntos x, y e z não foi realizada no presente artigo (ver Tabela 1).

As instruções, estímulos e tríades para complementação foram apresentados na tela de um computador (Arial, tamanho 60, em cor preta e em negrito sob fundo branco) utilizando o programa PowerPoint. O experimentador anotava as respostas dos sujeitos. O tempo de exposição das palavras durante as tarefas de codificação foi de $5 \mathrm{~s}$ e o das tríades para complementação, de $15 \mathrm{~s}$. A ordem seqüencial de realização das tarefas de codificação, bem como as listas de apresentação e recordação, foram balanceadas entre gêneros e, na medida do possível, entre sujeitos, já que o número de voluntários não permitia todas as combinações de listas em todas as condições de apresentação.

\section{Resultados}

A Tabela 2 traz as médias e desvios-padrão das proporções de tríades completadas com palavras-alvo de acordo com a codificação durante a apresentação e instrução do teste de complementação.

Para a aplicação do Critério de Recordação Intencional (CRI) realizou-se, primeiramente, uma Análise de Variância (ANOVA) de duas vias para a proporção de tríades completadas com palavras-alvo nos testes indireto e direto de inclusão, com os fatores codificação (3 níveis: perceptual, conceitual, ao acaso/não-familiares) e instrução do teste (2 níveis: indireto e direto). Verificou-se somente efeito de codificação $\left(\mathrm{F}_{2,22}=29,07 ; p<0,001\right)$, indicativo de efeitos de memória, isto é, menor proporção de complementações com palavras-alvo ao acaso que com palavras familiares codificadas de ambas as formas, como indicado pelo teste $t$ de Tukey (ps<0,001). Além disso, a codificação conceitual facilitou a complementação em relação à perceptual (Tukey: $p<0,03)$.

Tendo em vista que efeitos gerais de memória foram observados, uma segunda ANOVA foi realizada para comparar a complementação ao acaso após os dois tipos de instrução, indireta e direta. Como não houve efeito significante, foram empregadas ANOVAs considerando somente as palavras familiares para estudar os efeitos de codificação durante a apresentação e de possíveis interações dessa com a instrução do teste. Os fatores dessa análise foram codificação (perceptual e conceitual) e instrução (direta e indireta). Nessa análise foi observado efeito de codificação $\left(\mathrm{F}_{1,11}=12,06 ; p<0,006\right)$, tendo as palavras processadas conceitualmente sido mais completadas que as palavras aprendidas de forma perceptual (Tukey: $p<0,006)$. Houve também interação de codificação e instrução $\left(\mathrm{F}_{1,11}=6,24\right.$; $p<0,03)$, mostrando que as palavras codificadas conceitualmente no teste direto levaram a mais complementações do que palavras codificadas perceptualmente em ambos os testes (direto e indireto) (Tukey: $p<0,009$ ). Além disso, as palavras codificadas conceitualmente no teste direto produziram uma tendência para mais complementações após instrução direta do que após a indireta (Tukey: tendência, $p=0,09$ ). Em contraste, foi indiferenciada a complementação de palavras codificadas perceptual e conceitualmente no teste indireto, bem como a complementação em ambos os testes após codificação perceptual.

Uma ANOVA alternativa foi realizada subtraindo o desempenho ao acaso médio das proporções de palavras familiares (processadas perceptual e conceitualmente), novamente com codificação (perceptual e conceitual) e instrução (direta e indireta) como fatores. Os mesmos efeitos foram verificados. 
Tabela 1

Exemplo dos Conjuntos de Palavras (Representados por Letras) Envolvidos nas Tarefas de Apresentação e Recordação para um Participante

\begin{tabular}{cccc}
\hline & \multicolumn{3}{c}{ PROCESSAMENTO } \\
\cline { 2 - 4 } & Concept. & Percep. & ausente (acaso) \\
\hline Apresentação & ABx & CDy & - \\
Recordação & A & C & E \\
& Indireta & B & D
\end{tabular}

Nota.: O participante que realizava a tarefa de apreciação com a lis ta que incluía os conjuntos de palavras A e B tinha a recordação de estímulos codificados conceptualmente mensurada através da proporção de estímulos do conjunto A completada na tarefa indireta e do conjunto $\mathrm{B}$, na tarefa direta. A recordação dos estímulos dos conjuntos $\mathrm{C}$ e D apresentados na tarefa de contagem de espaços (codificação perceptiva) foi avaliada pela complementação das palavras desses conjuntos nas respectivas tarefas indireta e direta. Para determinar a probabilidade ao acaso de que palavras-alvo fossem completadas, utilizou-se as complementações das tríades referentes aos conjuntos $\mathrm{E}$ e $\mathrm{F}$ que não tinham sido apresentadas a esse sujeito. A complementação de palavras dos conjuntos x, y e z não foi avaliada (ver texto).

\section{Tabela 2}

Proporção (Média $\pm D P)$ de Tríades Completadas com Palavras-Alvo de acordo com a Tarefa de Codificação durante a Apresentação e a Instrução do Teste de Evocação

\begin{tabular}{cccc}
\hline Teste & \multicolumn{3}{c}{ Codificação } \\
\hline \multirow{3}{*}{ Indireta } & Conceitual & Perceptual & Acaso \\
\cline { 2 - 4 } Direta & $0,68 \pm 0,11$ & $0,61 \pm 0,11$ & $0,37 \pm 0,15$ \\
$0,76 \pm 0,17$ & $0,58 \pm 0,17$ & $0,43 \pm 0,14$ \\
\hline
\end{tabular}

Nota.: * Com teste direto palavras codificadas conceitualmente foram usadas para completar tríades mais freqüentemente que as codificadas perceptualmente nos testes indireto e direto e tenderam a ser mais usadas que palavras codificadas perceptualmente no teste indireto (ver texto).

\section{Discussão}

A aplicação do Critério de Recordação Intencional (CRI) aos resultados obtidos nos testes de complementação indireta e direta de tríades do presente trabalho mostra as predições dos autores dessa metodologia (ver Bishop \& Curran, 1995; Pompéia, 2000; Schacter et al., 1989): em primeiro lugar, foi observado que a apresentação de palavras, seja após codificação perceptual ou conceitual, elevou a probabilidade de complementação de tríades com essas palavras quando comparada à complementação ao acaso, ou seja, foi possível demonstrar que houve memória para esses estímulos. A comparação das respostas em ambos os testes indireto e direto (que diferiram somente na instrução do teste de recordação) mostrou que a estratégia de memória empregada no teste direto foi preponderantemente de memória explícita/ episódica, pois foi verificada facilitação na complementação após codificação conceitual, tipo de processamento que aumenta o uso desse tipo de memória (Blaxton, 1989; Richardson-Klavehn \& Bjork, 1988; Roediger, 1990). Ao contrário, o uso de informações aprendidas de forma perceptual não foi alterado pela instrução, direta ou indireta, o que também está de acordo com a literatura (RichardsonKlavehn \& Bjork, 1988; Roediger, 1990). Foi possível determinar também que não houve contaminação expressiva de memória explícita na complementação do teste indireto, pois não foi verificado efeito diferencial de codificação após esse teste. Além disso, a complementação conceitual tendeu a aumentar a complementação no teste direto quando comparada ao indireto, mostrando que a intenção de recordar informações leva a maiores índices de recordação de memória explícita. Esses resultados foram verificados em dois tipos de análise, uma que considerou somente a complementação com palavras familiares e outra em que a complementação ao acaso foi subtraída da complementação com as palavras familiares, reforçando os achados.
Os resultados aqui obtidos mostraram que, a princípio, a aplicação do CRI aos resultados de testes de complementação de tríades (utilizando estímulos adequados para o português do Brasil) é vantajosa em relação à simples comparação de perfis de complementação em testes de avaliação de memória explícita e implícita distintos. Essa superioridade empírica permite determinar fatores subjacentes aos subtipos de memória de longo prazo envolvidos na realização do teste de complementação, especialmente porque a observância desse paradigma permite determinar diferenças estocásticas entre memória explícita e implícita. Ademais, o padrão de dissociações e efeitos paralelos observados para o desempenho nos testes que só diferem quanto à instrução pode gerar indícios de inter-relação entre subtipos de memória, além de mostrar possível dissociação de memória explícita e implícita após diversos tipos de manipulação, como o uso de drogas que alteram a memória, divisão de atenção, efeitos de idade e escolaridade, ou mesmo para investigar processos mnemônicos em populações de pacientes com déficits cognitivos.

O CRI, porém, não leva em consideração a ocorrência de “recordação involuntária” de memória explícita, isto é, a evocação implícita/automática de uma informação que é reconhecida "conscientemente" (ver Schacter et al., 1989; Richardson-Klavehn, Lee, Joubran \& Bjork, 1994). Não existem, contudo, métodos baseados em questionários que sejam confiáveis para determinar se essa estratégia foi de fato utilizada durante a evocação de estímulos (Toth, 2000). É importante lembrar, entretanto, que o CRI não considera a possibilidade de que, durante a realização dos testes de memória, os sujeitos se tornem "conscientes" ou percebam a relação entre o episódio de aprendizado e o teste de memória. O que, de fato, está implicado na utilização dessa metodologia é a presença ou ausência de volição, ou intenção de recordar informações. Todavia, deve-se ter em mente que, embora pareça claro que "consciência" de algo e "intenção" 
refletem processos distintos, a "consciência” é um prérequisito do controle intencional e, em muitos casos, parece encorajar tal controle (Toth, 2000). Portanto, a caracterização de testes em diretos e indiretos não elimina completamente a importância da determinação de como a "consciência" interfere na evocação de informações. Assim, apesar das evidentes vantagens metodológicas em se aplicar o CRI para dissociar memória explícita da implícita, a dissociação entre desempenho em testes diretos e indiretos de memória em alguns aspectos apresenta limitações similares à abordagem clássica, isto é, não permite eliminar a possibilidade de que processos "conscientes" contaminem medidas de processos "não conscientes” e vice-versa, embora possibilite diagnosticar casos de contaminação considerável de memória explícita em testes indiretos, como ilustrado aqui.

\section{Referências}

Bishop, K.I. \& Curran, H.V. (1995). Psychopharmacological analysis of implicit and explicit memory: a study with lorazepam and the benzodiazepine antagonist flumazenil. Psychopharmacology, 40, 267-278.

Blaxton, T.A. (1989). Investigating dissociations among memory measures: support for transfer-appropriate processing framework. Journal of Experimental Psychology: Learning, Memory and Cognition, 15, 657-668.

Gupo Coquetel (1983). Dicionário para as palavras cruzadas diretas: exclusivas do Grupo Coquetel. Rio de janeiro: Tecnoprint.

Erdelyi, M.H. (1992). Psychodynamics and the unconscious. American Psychologist, 47, 784-787.

Farthing, G.W. (1992). The psychology of consciousness. New Jearsey, Englewood Cliffs: Prendice Hall.

Gershberg, F.B. \& Shimamura, A.P. (1994). Serial position effects in implicit and explicit tests of memory. Journal of Experimental Psychology: Learning, Memory and Cognition, 20, 1370-1378.

Graf, P. \& Schacter, D.L. (1985). Implicit and explicit memory for new associations in normal and amnesic subjects. Journal of Experimental Psychology: Learning, Memory and Cognition, 11, 501-518.

Graf, P. \& Williams, D. (1987). Completion norms of 40 threeletter word stems. Behavioral Research Methods, Instruments and Computers, 19, 422-445.

Greenwald, A.G. (1992). New look 3: unconscious cognition reclaimed. American Psychologist, 47, 766-779.

Jacoby, L.L. (1998). Invariance in automatic influences of memory: towards a user's guide for the process dissociation procedure. Journal of Experimental Psychology: Learning, Memory and Cognition, 24, 3-26.

Jacoby, L.L., Lindsay, D.S., \& Toth, J.P. (1992). Unconscious influences revealed: attention, awareness, and control. American Psychologist, 47, 802-809.

Johnson, M.K. \& Hasher, L. (1987). Human learning and memory. Annual Review of Psychology, 38, 631-668.

Kandel, E.R. (1999). Biology and the future of psychoanalysis: a new intellectual framework for psychiatry revisited. American Journal of Psychiatry, 156, 505-524.

Kihlstrom, J.F., Barnhardt, T.M., \& Tataryn, D.J. (1992). The psychological unconscious: found, lost, and regained. American Psychologist, 47, 788-791.
Kihlstrom, J.F (1995). The rediscovery of the unconscious. In H. Morowitz \& J. Singer (Eds.), The mind and brain and complex adaptation systems. SFI Studies in the sciences of complexity (Vol. XXII). New York: Addison-Wesley.

Kihlstrom, J.F. (1987). The cognitive unconscious. Science, 237, $1445-1452$.

Magila, M.C. \& Xavier, G.F. (1999). Modelos de memória de longa duração em humanos. Psicologia: Teoria e Pesquisa, 15, $37-44$.

Moscovitch, M. (2000). Theories of memory and consciousness. In E. Tulving \& F.I.M. Craik (Eds.), The handbook of memory (pp.609-626). Oxford: Oxford University Press.

Pompéia, S. (2000). Benzodiazepinicos e cognição: efeitos típicos e atípicos em voluntários normais. Tese de Doutorado não publicada, Departamento de Psicobiologia da Universidade Federal de São Paulo. São Paulo, SP.

Pompéia, S. \& Bueno, O.F.A. (1998). Preliminary adaptation into Portuguese of a standerdised picture set for use in research and neuropsychological assessment. Arquivos de Neuropsiquiatria, 56, 366-374.

Pompéia, S., Paes, A.T., \& Bueno, O.F.A. (2003). Teste de complementação de letras: estímulos para uso no Brasil. Psicologia: Teoria e Pesquisa, 19, 65-73.

Richardson-Klavehn, A. \& Bjork, R.A. (1988). Measures of memory. Annual Review of Psychology, 39, 475-543.

Richardson-Klavehn, A., Lee, M.G., Joubran, R., \& Bjork, R.A. (1994). Intention and awareness in perceptual identification priming. Memory and Cognition. 22, 293-312.

Roediger, H.L. (1990). Implicit memory: retention without remembering. American Psychologist, 45, 1043-1056.

Schacter, D.L. (1992). Understanding implicit memory: a cognitive neuroscience approach. American Psychologist, 47, 559-569.

Schacter, D.L., Bowers J.S., \& Booker J. (1989). Intention, awareness and implicit memory, the retrieval intentionality criterion. In S. Lewandowsky, J.C. Dunn \& K. Kirsner (Eds.), Implicit memory: theoretical issues (pp.47-64). Hillsdale: Lawrence Erlbaum.

Toth, J.P. (2000). Nonconscious forms of human memory. In E. Tulving \& F.I.M. Craik (Eds.), The handbook of memory (pp.245-266). Oxford: Oxford University Press.

Toth, J.P., Lindsay, S., \& Jacoby, L.L. (1992). Awareness, automaticity, and memory dissociations. In L.R. Squire \& N. Butters (Eds.), The neuropsychology of memory (pp.46-57). New York: Guilford Press. 


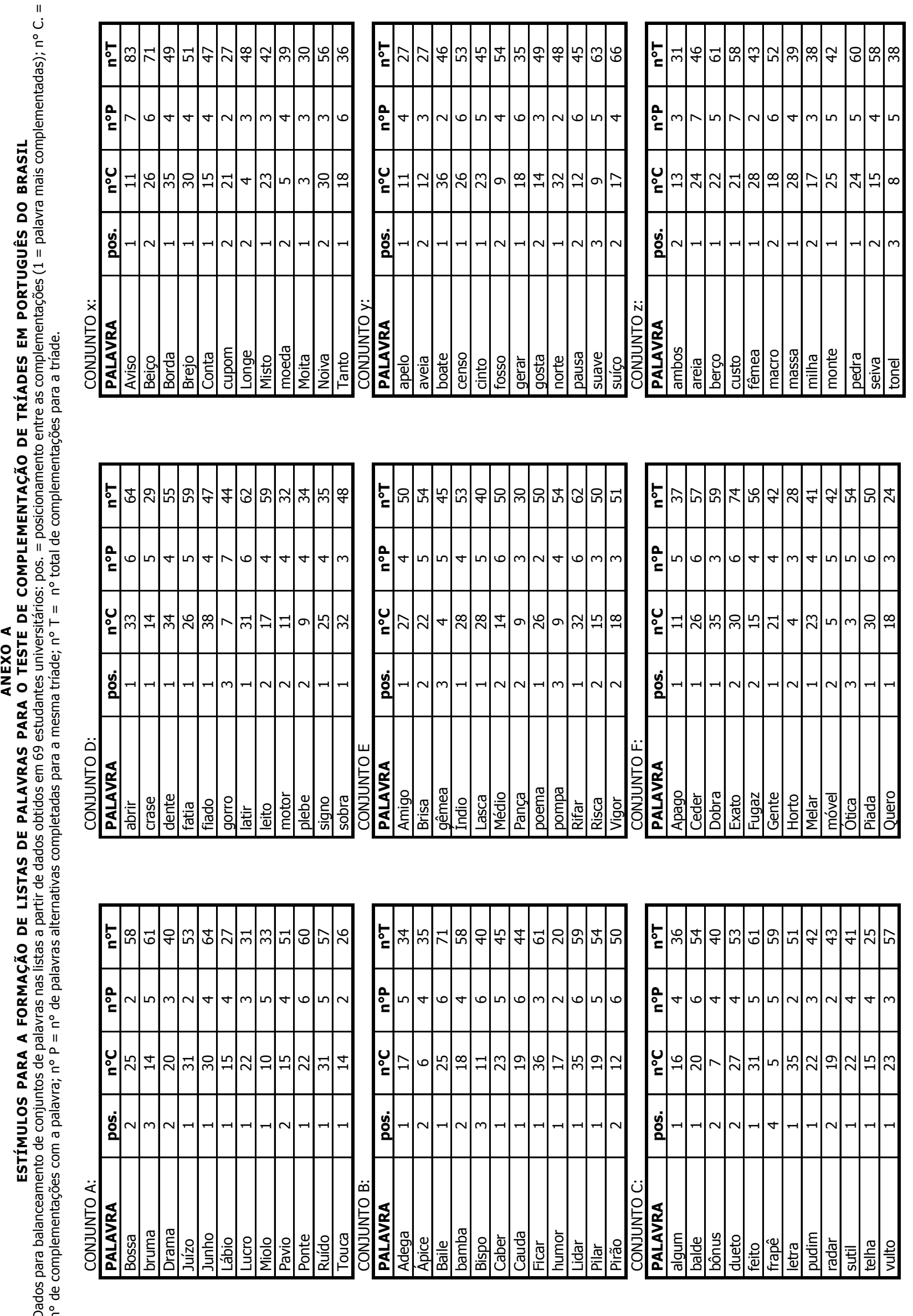

